\title{
A cone-plate apparatus for the in vitro biochemical and molecular analysis of the effect of shear stress on adherent cells
}

\author{
Adel M. Malek ${ }^{1}$, Richard Ahlquist ${ }^{1}$, Gary H. Gibbons ${ }^{2}$, Victor J. Dzau² \& Seigo Izumo ${ }^{3}$ \\ 'Department of Neurosurgery, Brigham \& Women's Hospital/Children's Hospital and Harvard Medical School, \\ Boston, MA; ${ }^{2}$ Falk Cardiovascular Center, Stanford University Medical School, Stanford, CA; ${ }^{3}$ Cardiovascular \\ Research Center, University of Michigan, Ann Arbor, MI, USA
}

Accepted in revised form 11 April 1995

\begin{abstract}
Living cells are constantly exposed to a variety of complex mechanical stimuli which are thought to be critical in the control of tissue structure and function. Endothelial and smooth muscle cells in the blood vessel are ideal candidates for the study of blood flow-induced cellular regulation. We describe here a cone-plate viscometer apparatus which is specially-designed for studying the effect of fluid shear stress on large populations of adherent cells in vitro. Using conventional polystyrene tissue culture plates, the apparatus is self-contained, fits inside a standard tissue culture incubator, and provides $75-150 \mathrm{~cm}^{2}$ of useful surface area for cell growth. This capability makes it ideal for studying gene regulation using Northern analysis, nuclear
\end{abstract}

runoff transcription, transfection with reporter constructs, as well as immunochemical staining. The closed-volume design of the device is also wellsuited for isotopic labelling, pharmacological studies, and for the detection of minute amounts of secreted cell products. The setup allows the use of either steady, time- and direction-varying laminar, or turbulent shear stress. We provide a detailed assembly procedure and review the method for computing shear stress magnitude and Reynolds number. Ink flow analysis, dynamic response characterization, and LDH measurements are presented to confirm the device's fluid mechanical properties and demonstrate the absence of cell injury.

Key words: Biomechanics, Cell culture, Endothelium, Mechanotransduction, Mechanical stress, Scientific instruments, Rheology

Abbreviations: BAE: bovine aortic endothelial; LDH: lactic dehydrogenase; TCP: tissue culture plate; UHMW: ultra-high molecular weight

\section{Introduction}

There has recently been a resurgence of interest in the effect of biomechanical forces, specifically fluid shear stress, tensile strain, and hydrostatic pressure, on the structure and function of various cell types in the cardiovascular system including endothelium $[1,2]$, smooth muscle [3] and cardiac myocyte [4]. This interest has grown from observations which implicate the ever-present mechanical forces in determining tissue and organ growth and development [5]. In the blood vessel, fluid shear stress, the force acting on the surface of the endothelium as a result of blood flow, has been clearly demonstrated to affect the morphology $[6,7]$ and functional phenotype of the endothelial cell [8-10].

The importance of the local fluid shear forces in determining vascular structure has been illustrated in numerous studies in vivo $[11,12]$. In addition, local fluid forces may play a role in the development of atherosclerosis, particularly around vessel bifurca- tions and in regions of flow separation where shear stress magnitude is low and time-varying [13, 14]. The above findings have encouraged the characterization of the endothelial response to fluid shear stress in vitro at multiple levels, including second messenger signalling [15-17], generation of functional mediators [18], morphology [6, 19, 20], and regulation of gene expression $[1,2]$.

Although a number of devices have been described for the study of the effect of shear stress on endothelial function in vitro $[20,21]$, they have suffered from limitations that have curtailed their use as a general multi-purpose shear stress environment. Some have employed a small surface area for culture of cells on microscope cover slides [22], while others have been unsuitable for adherent cells [23], necessitated time-consuming assembly and disassembly procedures, or required the connection to closed circuit systems involving tubing and pump mechanisms [24]. These shortcomings have made it difficult to readily use biochemical and molecular assays 
requiring large cell sample size, such as nuclearrunoff transcription, Northern analysis, or protein immunoprecipitation. Furthermore, the use of pumps and tubing in the case of parallel-plate [18] or capillary tube $[25,26]$ systems entails a large volume of circulating culture medium which renders radiolabelling and pharmacological experiments cumbersome, and could potentially introduce a hydrostatic pressure artifact [27]. Since fluid shear stress in vivo exhibits a spectrum of differing dynamic characteristics, which are known to influence cellular function $[10,28,29]$, it is deemed important for the apparatus to readily reproduce pulsatile, reversing or turbulent shear stress in the final design.

The apparatus presented in this paper is based on the established cone-plate viscometer geometry which was originally proposed and used in tissue culture of BAE cells by Dewey and colleagues [30]. Using the cone-plate device in pioneering experiments they were able to duplicate the BAE cell alignment seen in vivo in response to flow [6] and demonstrate the importance of the dynamic characteristics of the flow stimulus in affecting cellular function [28]. Similarly Schnittler et al. used a coneplate type of apparatus $[7,31]$ to study endothelial cell morphology response to fluid shear stress. Both of these devices employed a complex positioning mechanism and used small surface area for cell growth, rendering their use for biochemical studies suboptimal. Yoshizumi et al. [32] relied on a coneplate viscometer with a small $5-\mathrm{cm}$ tissue culture plate for the study of flow effects on endothelin-1 regulation. The latter group, however, did not describe the design nor the method of construction and testing.

The subject of the present report is a cone-plate viscometer which was designed from the outset to achieve the following specific goals:

1. enable the use of commercially-available tissue culture plates to avoid costly specialized culture vessels,

2. allow compatibility with standard tissue culture methods and require minimal additional specialpurpose equipment such as tubing or pumps,

3. provide high experimental throughput and quick isolation of media and cell samples through a quick and simple disassembly/reassembly process,

4. offer a large cell growth surface to obtain a sufficient number of cells for protein, mRNA and transcriptional studies while minimizing the need for pooling of multiple samples or PCR amplification procedures.

We describe here the detailed design and step-by-step method of construction and testing of a cone-plate viscometer apparatus which is optimized for the study of shear stress effects on adherent cells in a wide range of experimental applications.

\section{Materials}

A. Equipment and raw materials

1. DC motor and gearbox model E352.

2. C motor controller.

3. Stainless steel 304 precision-ground shaft. ${ }^{2}$

4. Flexible coupling. ${ }^{2}$

5. Aluminum alloy stock. ${ }^{3}$

6. UHMW polyethylene stock. ${ }^{4}$

7. Plexiglass ${ }^{\mathrm{TM}}$ stock. $^{4}$

8. Ultra-high precision ball bearing. ${ }^{5}$

9. Digital-to-Analogy (D/A) converter broad model DT2901. ${ }^{6}$

10. Storage oscilloscope model Hitachi VC6025.

11. Electrical signal generator model NF FG$121 \mathrm{~B}^{8}$

12. Personal computer model IBM PS/2 55SX. ${ }^{9}$

13. Personal computer model Apple Macintosh. ${ }^{10}$

14. Water-jacketed tissue culture incubator. ${ }^{11}$

15. Inverted phase-contrast microscope model Olympus IMT-2. ${ }^{12}$

16. Epifluorescence microscope model Olympus BH-2. ${ }^{12}$

B. Supplies and software

1. Tissue culture plate $10 \mathrm{~cm}$ (Falcon, No. 3003 ) and $15 \mathrm{~cm}$ (Falcon, No. 3025) diameter. $^{13}$

2. Transwell tissue culture plate $10 \mathrm{~cm}$ with $0.4 \mu \mathrm{M}$ pore size polycarbonate filter insert (Costar, No. 3419). ${ }^{14}$

3. Microscope slide cover (Fisher, No. 12 546). ${ }^{15}$

4. Canon-Fenske type viscometer (Fisher, No. $13-617 \mathrm{C}) .{ }^{15}$

5. Silicone adhesive (Fisher, No. 04-7695). ${ }^{16}$

6. MathCad computation software. ${ }^{17}$

C. Media and chemicals

1. Dulbecco's modification of Eagle's medium (DMEM) (Gibco-BRL, No. 31600). ${ }^{18}$

2. Calf serum (Gibco-BRL, No. 16170). ${ }^{19}$

3. Hepes buffer (Sigma, No. H7523). ${ }^{19}$

4. Penicillin G/Streptomycin sulfate. ${ }^{18}$

5. Acetylated low-density lipoprotein (AcLDL). ${ }^{20}$

6. Uncharged Dextran (M.W. 76,000) (Sigma, No. D1537). ${ }^{19}$

D. Tissue

Fresh bovine descending thoracic aortae. ${ }^{21}$

\section{Procedures}

\section{Construction and use of apparatus}

1. A plate of aluminum alloy is machined to serve as a base for the DC-motor and gearbox and accept either a 10 or $15 \mathrm{~cm}$ dia. tissue culture plate (TCP) in a rimmed depression as shown in Figure 1.

2. An oval-shaped opening is drilled through the 
A

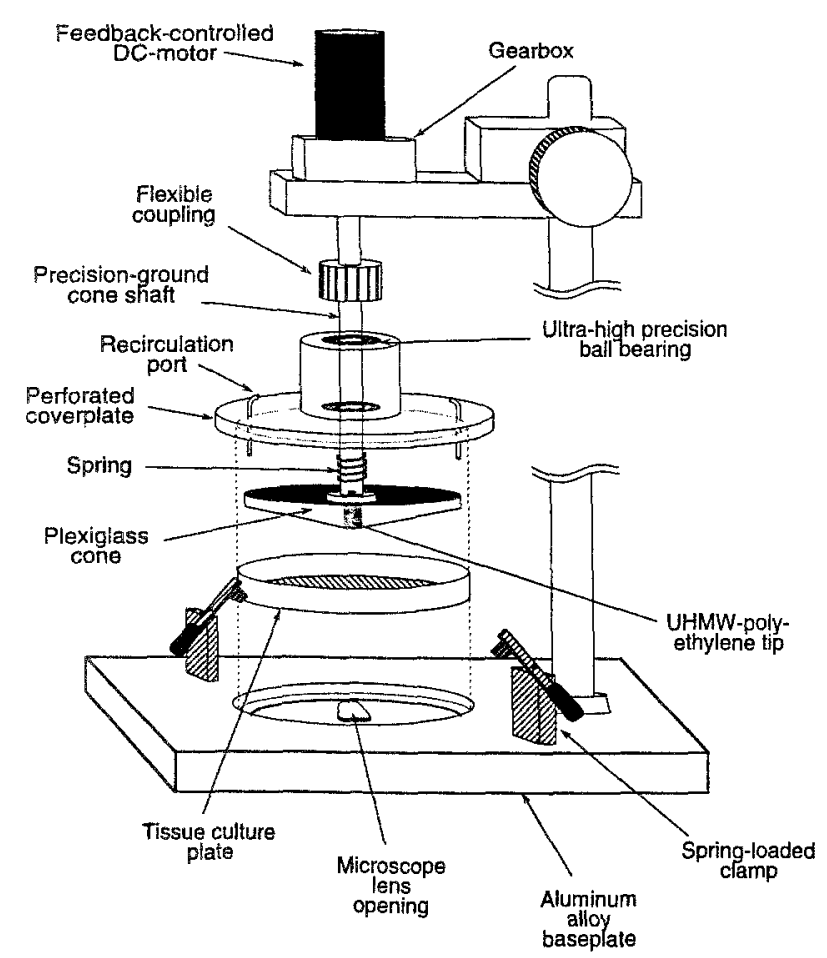

B

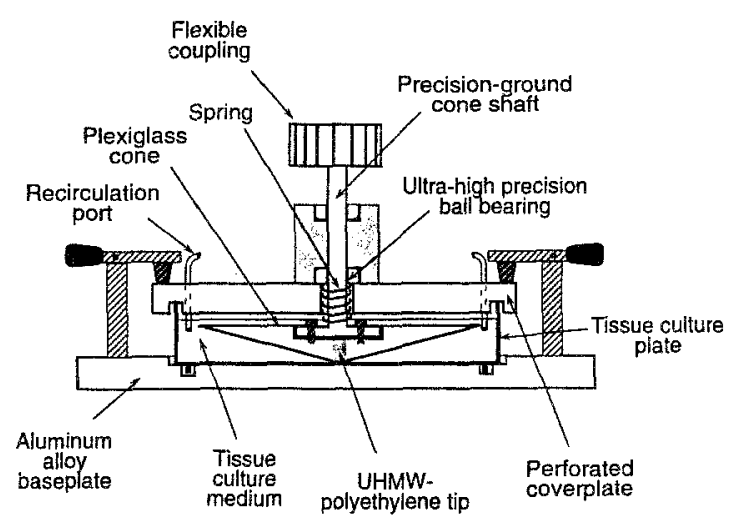

C

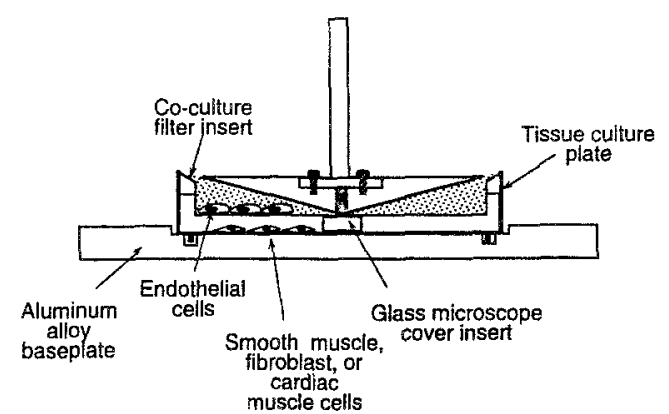

Figure 1. (A) Assembly drawing of proposed design illustrating the use of a standard tissue culture plate resting on a flat aluminum base-plate and a concentric plexiglas ${ }^{\mathrm{TM}}$ cone impinging on its center tip of ultra-high molecular weight (UHMW) polyethylene via a spring load. The cone is connected via a flexible coupling to a feedback-controlled DCmotor that allows the setting and maintenance of the rotational velocity $\omega$. The cone shaft slides freely via an interference fit through two aligned ultra-high precision ball bearings connected to a clear coverplate held in place by spring-loaded clamps. The coverplate incorporates aeration holes for gas exchange with the external environment of the tissue culture incubator, and two ports for sampling or recirculating flow medium during device operation, or for controlling the gas composition overlying the tissue culture medium.

(B) Side view illustrating the relative positions of the main components of the cone-plate viscometer. Note the tissue culture medium present between the tissue culture plate and the cone. The use of quick-release spring loaded clamps and low-tolerance machining allows swift change of tissue culture plate under sterile conditions, thereby enabling a high experimental throughput. Operation can be terminated and sample harvested in $\sim 5 \mathrm{~s}$, and exchanged for another sample within $\sim 15 \mathrm{~s}$.

(C) Schematic diagram of modified cone-plate viscometer, designed specifically for use with co-culture plates, whereby endothelial cells are grown on the filter insert resting at its center on a microscope slide cover affixed to the bottom plate. Smooth muscle, cardiac muscle or fibroblast cells can be grown on the bottom surface, thereby allowing the abluminal transport of substances derived from endothelium exposed to fluid shear stress.

surface of the TCP base to permit the fitting of the apparatus on a microscope storage (Olympus IMT-2) and provide for the on-line visualization of the monolayer, thereby allowing the study in real time of fluid shear stress on cell shape and intracellular $\mathrm{pH}$.

3. A precision-ground $3 / 8^{\prime \prime}$ (for $10 \mathrm{~cm} \mathrm{TCP)} \mathrm{and}$ $3 / 4^{\prime \prime}$ (for $15 \mathrm{~cm}$ TCP) stainless steel shaft is connected to a block of transparent Plexiglas ${ }^{\mathrm{TM}}$. The block of Plexiglas ${ }^{\mathrm{TM}}$ is then machined to provide a conical surface of angle between $0.5^{\circ}$ and $5^{\circ}$. Careful micromeasurements are made of the cone surface as it is turned in the highprecision lathe to insure less than $\pm 0.002^{\prime \prime}$ of wobble at the outer radius. A center plug of UHMW polyethylene is inserted and machined into the tip of the cone, which is then polished to optical clarity.

4. The cone shaft is inserted by a slight friction fit into a pair of ultra high precision ball bearings housed in an aluminum alloy cylindrical block. A stainless steel spring is inserted around the cone shaft to act in compression against the inner race of the ball bearing and the shaft base.

5. The bearing housing is permanently secured by 6 screws to a transparent Plexiglas ${ }^{\mathrm{TM}}$ coverplate containing a groove designed to accept the lip of the tissue culture dish at a $90^{\circ}$ angle. Holes are drilled in the coverplate to allow gas exchange between the sample and the outside atmosphere and enable the use of the device inside a standard tissue-culture incubator $\left(37^{\circ} \mathrm{C}\right.$, 
$5 \% \mathrm{CO}_{2}$ ). Two ports are installed in the cover plate to allow sampling and recirculation of medium during operation where needed. The recirculation ports can also be used to control the gas composition overlying the tissue culture medium by sealing the remaining aeration holes in the coverplate with silicon adhesive.

6. The cone shaft is connected via a flexible coupling (Figure 1A) to a DC-motor with a feedback controller that insures accurate speed control over long periods of time. When the coverplate is in position and is secured via the spring-loaded clamps, the device appears as shown in cross-section (Figure 1B).

7. The DC-motor controller is connected to accept inputs from an external signal generator or computer output via a digital to analog $(\mathrm{D} / \mathrm{A})$ board, which allows for specific time profiles of cone rotational velocity. The final product using a $15 \mathrm{~cm}$ TCP and the $3 / 4^{\prime \prime}$ cone shaft is shown in Figure 2. The size and type of design allows the use of up to four devices simultaneously in a standard tissue culture incubator. This enables a high experimental throughput since the four samples can be simultaneously subjected to differing fluid shear stress conditions.

8. A version of the device is built to accommodate co-culture of two cell types by using $10 \mathrm{~cm}$ tissue culture plates with filter inserts (Figure 1C). This device uses a thinner cone of smaller radius, which rests on the filter insert, and in order to maintain the flexible filter surface flat, on a glass microscope slide cover secured on the center of the bottom plate with silicone adhesive. This version enables the user to co-culture endothelial cells plated on the filter insert and exposed to shear stress, with stationary smooth muscle, fibroblast, or cardiac cells plated on the lower tissue culture plate surface.

9. The dynamic characteristics of the device are determined by using either a Model FG-121B signal generator or a Model DT2901 D/A board connected to an IBM PS/2 55SX personal computer to drive the motor controller. The cone rotation velocity signal is acquired by measuring the DC-motor tachometer output with a Hitachi

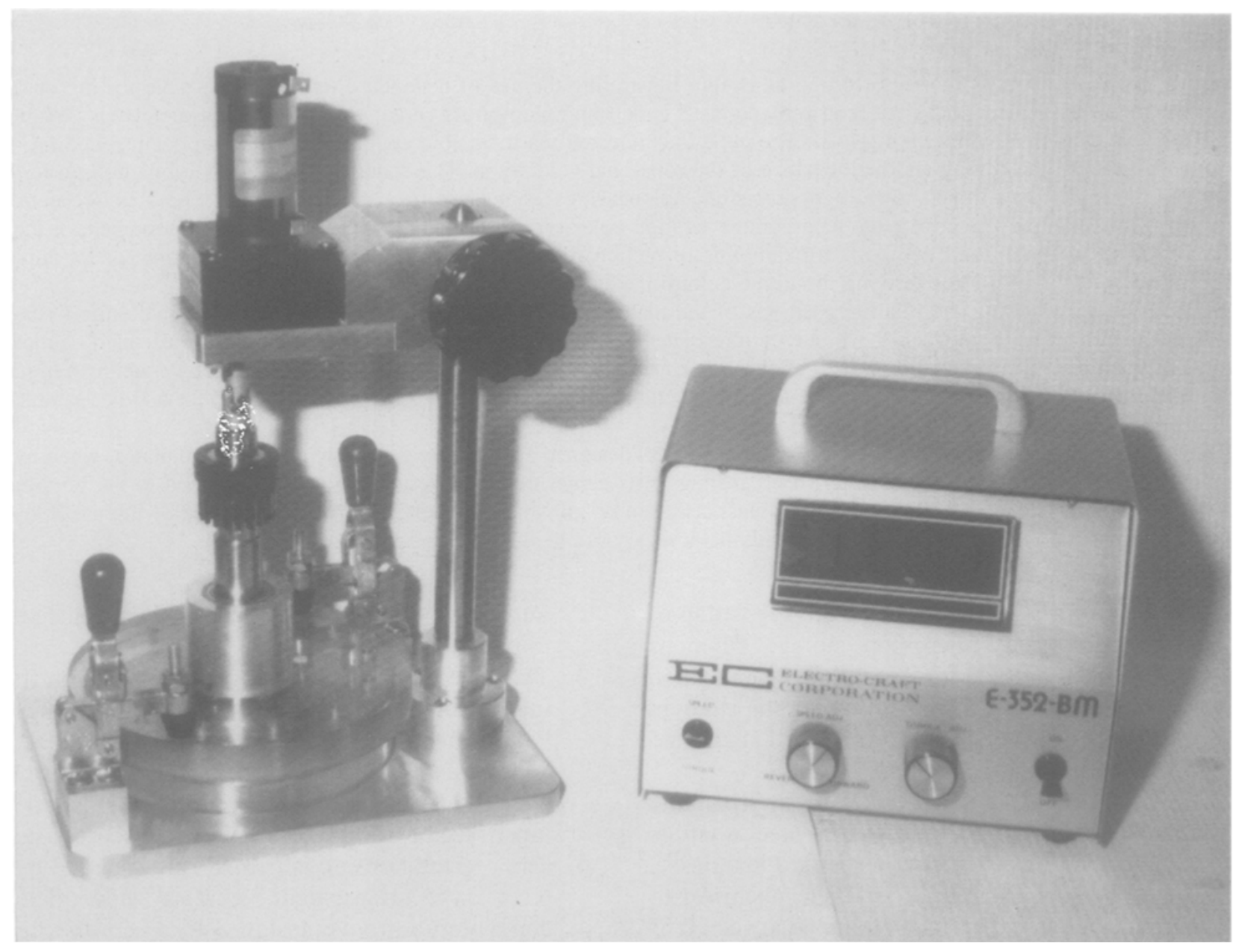

Figure 2. Photograph of actual apparatus using a $15 \mathrm{~cm}$ tissue culture plate (Falcon, No. 3025) with a $3 / 4^{\prime \prime}$ cone shaft and matching motor controller. The same baseplate can be also used with a $10 \mathrm{~cm}$ plate (Falcon model, No. 3003) thanks to an additional flush concentric groove for proper TCP centering. The compact design of the apparatus allows the simultaneous use of up to four devices in a standard water-jacketed tissue culture incubator, enabling high experimental throughput. 
model VC6025 storage oscilloscope. For dynamic frequency response analysis, a sinusoidal velocity signal with peak rotational velocity of either $3 \mathrm{rev} / \mathrm{s}$ or $6 \mathrm{rev} / \mathrm{s}$ serves as the input to the motor controller while the tachometer signal is acquired using the storage oscilloscope and the magnitude of the peak velocity is recorded. A gain value, the ratio of the actual velocity to that of the input signal, was measured at the following frequencies of the sine wave stimulus: $0.1,0.5,1-10,15$, and 20 $\mathrm{Hz}$.

10. Kinematic viscosity of the medium is measured using a calibrated Canon-Fenske type viscometer. Different combinations of cone angle $\left(0.5-5^{\circ}\right)$ and rotational velocities are chosen to achieve the different flow regimes described below. Shear stress magnitude, $\tau_{\mathrm{ss}}$, and Reynolds number, $R_{c}$, are computed by using relations previously derived by Sdougos et al. [30, 33] (Figure 3) in a MathCad routine which is presented in Figure 4 running on a Macintosh computer.

11. The coverplate, attached bearing housing, and cone are washed in laboratory detergent, avoiding alcohol to prevent damage to the polished Plexiglas ${ }^{\mathrm{TM}}$ surface, then thoroughly rinsed with deionized water and exposed to 30 min of ultraviolet irradiation for sterilization.

12. Bovine aortic endothelial (BAE) cells are harvested from descending thoracic aortae obtained from the local abattoir by collagenase digestion [34], and are used between passages 6-15.
Purity of the isolated cells is confirmed using the acetylated-LDL uptake method [35]. The cells are grown in Dulbecco's modified Eagle (DME) medium supplemented with $10 \%$ calf serum, 4 $\mathrm{mM}$ L-glutamine, $25 \mathrm{mM}$ Hepes $\mathrm{pH} 7.4,10$ units $/ \mathrm{ml}$ of penicillin, and $10 \mu \mathrm{g} / \mathrm{ml}$ of streptomycin, at $37{ }^{\circ} \mathrm{C}, 5 \% \quad \mathrm{CO}_{2}$ in a humidified incubator. Lactic dehydrogenase (LDH) concentrations are determined using an automated analyzer.

13. Under sterile conditions with a laminar air flow hood, confluent BAE monolayers, last fed with complete growth medium or serum-free DME 24-48 hours prior to onset of flow, and grown in 10 or $15 \mathrm{~cm}$ TCP are mounted inside the pre-warmed cone-coverplate section of the apparatus. The coverplate, cone and underlying tissue culture plate are then transferred to the baseplate section housed inside the tissue culture incubator and secured in position with the clamps (Figure $1 \mathrm{~A})$. The cone is then connected to the DCmotor via the flexible coupling following proper alignment of the motor and gearbox assembly with the cone shaft.

\section{Method of computation of shear stress in cone- plate geometry}

Figure 3 shows the basic geometry of the cone of shallow angle $\alpha$ and maximum radius $r_{0}$ in contact at its tip with the flat plate being separated from it by tissue culture medium of viscosity $\mu$ and density $\rho(\nu=\mu / \rho)$. Previous work [30] has identified the existence of various modes of operation of this

$\begin{array}{lll}\alpha & = & \text { Angle of Cone } \\ \omega & = & \text { Angular Velocity } \\ \mu & = & \text { Viscosity } \\ \nu & = & \text { Kinematic Viscosity } \\ \mathrm{R}_{\mathrm{e}}= & \text { Reynolds Number } \\ r & = & \text { Radius } \\ \tau_{\mathrm{ss}} & = & \text { Shear Stress }\end{array}$

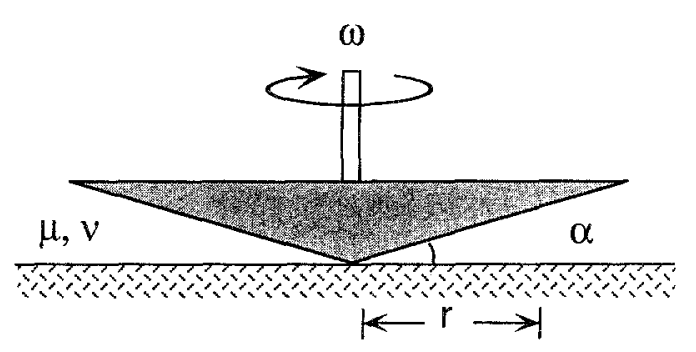

$$
\begin{aligned}
& \text { Eq. } 1 \quad R_{e}=\frac{r^{2} \omega \alpha^{2}}{12 v} \\
& \text { Eq. } 2\left|\tau_{\mathrm{ss}}\right| \cong\left(\frac{\mu \omega}{\alpha}\right)\left[1+2.58\left(\frac{R_{e}^{\frac{3}{2}}}{3.5+R_{e}}\right)-0.86\left(\frac{R_{e}^{\frac{5}{2}}}{\left(3.5+R_{e}\right)^{2}}\right)\right]
\end{aligned}
$$

Figure 3. Schematic description of a cone-plate viscometer device depicting a cone of angle $\alpha$ and radius $r_{0}$ rotating at $\omega * 2 \pi \mathrm{rad} / \mathrm{s}$ in a medium of static viscosity $\mu$ and kinematic viscosity $v$. The Reynolds number $R_{e}$ (Equation 1$)$ and the absolute magnitude of the shear stress $\tau_{\mathrm{ss}}$ (Equation 2), applied on the surface of the plate at a given radius $\mathrm{r}$, are computed as described by Sdougos et al. [30]. Note that $\tau_{\mathrm{ss}}$ tends to be uniform and independent of radius $r$ for very small values of $R_{e}\left(R_{e} \ll 0.1\right)$ while it shows significant dependence on the square root of $R$ at large values $\left(R_{e} \gg 1\right)$. Cells grown on the surface can be subjected to varying regimes of shear stress including laminar primary $\left(R_{e} \ll 0.1\right)$, secondary $(0.1$ $\left.<\mathrm{R}_{\mathrm{e}}<1 \sim 4\right)$ and turbulent shear stress $\left(\mathrm{R}_{e}>4\right)$ as described in the text. 
device determined largely by the Reynolds number, $\mathrm{R}_{\mathrm{e}}$ (Figure 3, Equation 1). At low values of $\mathrm{R}_{\mathrm{e}}\left(\mathrm{R}_{\mathrm{e}}<\right.$ 0.1 ), the system is in purely primary laminar fluid shear stress. The flow lines are the direction in which the shear stress acts at the surface of the plate are concentric circles. In addition, the shear stress magnitude is constant at all values of radius $r$. With increasing $R_{e}$ a secondary centripetal flow component is directed at the surface of the plate towards the tip of the cone $\left(0.1<R_{e}<1 \sim 4\right)$, causing the flow line at the plate to form an angle $\phi$ with respect to the tangent. At still higher levels of $R_{e}\left(R_{e}>4\right)$ shear stress at the surface becomes turbulent and progressively less well defined in time and space. For small cone angles $\left(\alpha \sim<5^{\circ}\right)$ it is possible to compute the Reynolds number, $R_{e}$, and the approximate shear stress absolute magnitude $\left|\tau_{\text {ss }}\right|$ at a given radius $r$ given the parameters by using the Equations 1 and 2 shown in Figure 3 [30]. For values of $R_{e}$ which are much greater than 4, Equation 2 becomes increasingly less accurate in estimating the true magnitude of the shear stress at the surface.

A routine written for MathCad, shown in Figure 4 , is used to compute the local value of shear stress, $\tau_{s s}$, and Reynolds number, $R_{e}$ at a given value $r$ of the radius as well as the profile of these values with respect to $r$. The average shear stress magnitude and Reynolds Number is obtained by weighted numerical integration (weight factor of $2 \pi \mathrm{r}$ ) of Equations 1 and 2. Figure 5 illustrates the profiles for three different sets of parameters. By proper choice of the parameters, it is possible to achieve steady laminar shear stress and turbulent shear stress of same average shear stress magnitude when integrating over the whole cell surface area. Medium viscosity, $\mu$, can be increased by adding uncharged dextran (MW 76,000) (up to 2.94 -fold at $5 \% \mathrm{wt} / \mathrm{vol}$ dextran) to the culture medium without affecting cell viability [ 22 and our unpublished observations].

\section{Results and discussion}

\section{Measurement of tissue culture plate convexity}

One of the central features of the presented design is the ability to use standard tissue culture plates. These plates, however, suffer from an inherent convexity in the center where the cone tip comes in

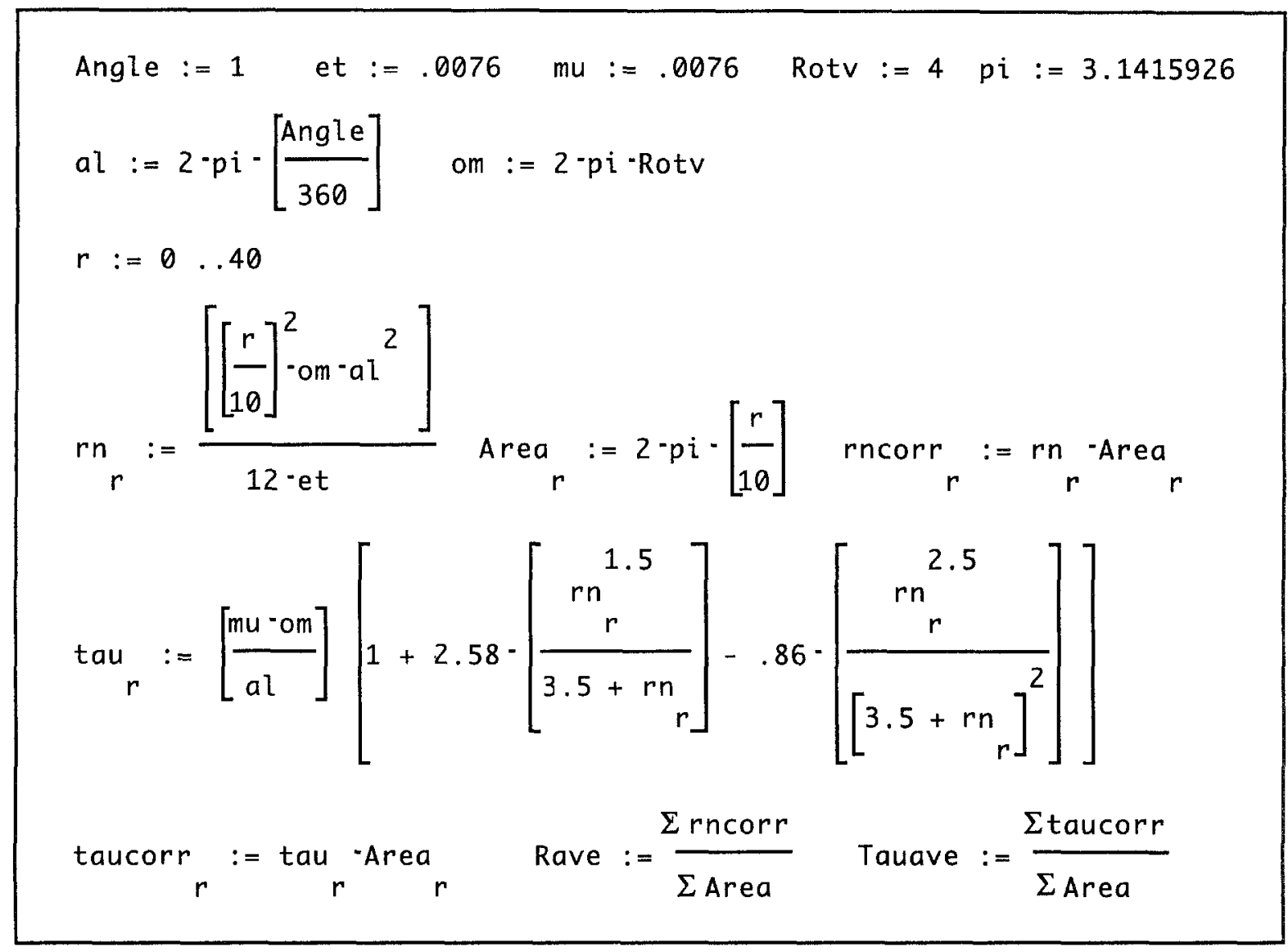

Figure 4. MathCAd routine used to compute $\tau_{\mathrm{ss}}$ and $\mathrm{R}_{\mathrm{e}}$ at various values of radius and average value per unit surface area. The average values of the Reynolds number and the shear stress magnitude per surface area are computed by numerical integration of $R_{c}$ and $\tau_{s s}$ (Equations $1 \& 2$, Figure 2) with respect to unit circumference, thereby obtaining a weighted average favoring values at large $\mathrm{r}$ (where the corresponding annular surface is proportional to $2 \pi \mathrm{r}$ ). Units are as follows: Angle (degrees), et (poise/ $\left.\mathrm{cm}^{3}\right) m u$ (poise), Rotv (rev/s), al (radians), om (radians/s), $r(\mathrm{~cm}), r n_{\mathrm{r}}$ and $r n c o r r_{\mathrm{r}}$ (unitless), Area $_{\mathrm{r}}\left(\mathrm{cm}^{2}\right)$, tau $_{\mathrm{r}}$ and taucorr $\mathrm{r}\left(\mathrm{dyn} / \mathrm{cm}^{2}\right)$. 


\begin{tabular}{llllll|}
$\alpha=5^{\circ}$ & $\omega=5^{\star} 2 \pi / s$ & $0 \%$ Dextran & $R_{\text {ave }}=21.5$ & $\tau_{\text {ave }}=21.4$ \\
$\cdots$ & $\alpha=1^{\circ}$ & $\omega=4^{*} 2 \pi / s$ & $0 \%$ Dextran & $R_{\text {ave }}=0.7$ & $\tau_{\text {ave }}=14.7$ \\
$\cdots \cdots$ & $\alpha=0.5^{\circ}$ & $\omega=3^{*} 2 \pi / s$ & $5 \%$ Dextran & $R_{\text {ave }}=0.04$ & $\tau_{\text {ave }}=49.0$ \\
\hline
\end{tabular}
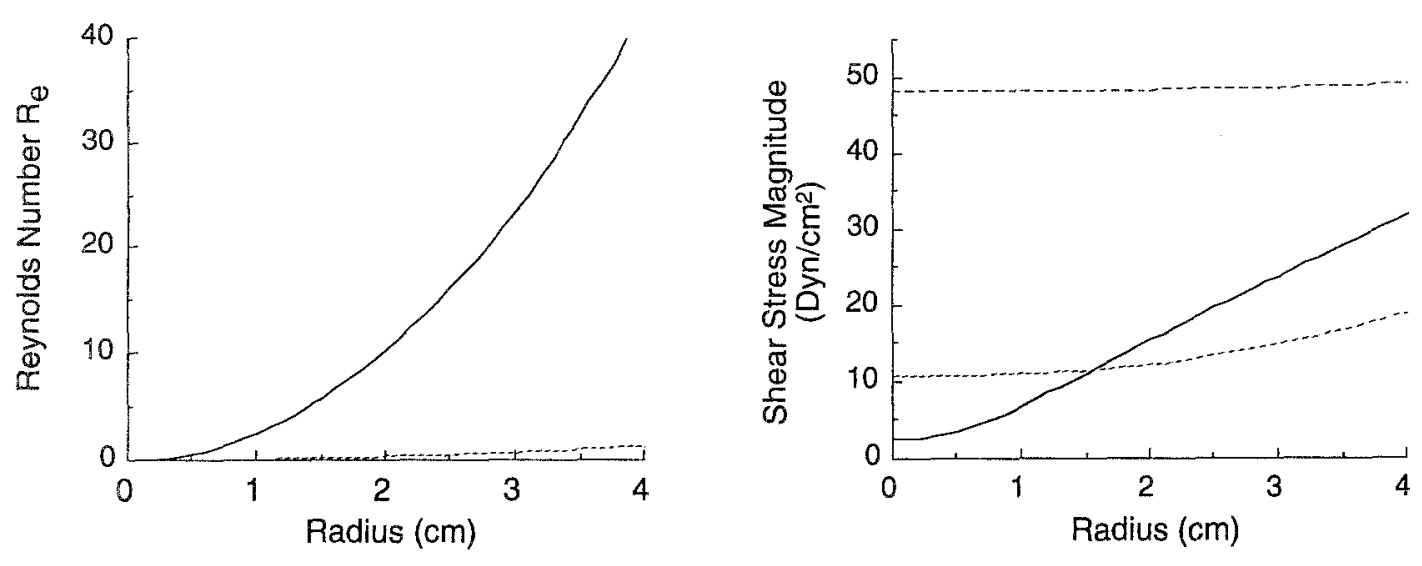

Figure 5. Typical profiles of Reynolds number, $\mathrm{R}_{\mathrm{e}}$, and absolute magnitude of shear stress, $\tau_{\mathrm{ss}}$, with respect to radius for three sets of parameters $(A, B, C)$ with different cone angle $\alpha$, rotational velocity $\omega$, and medium viscosity $\mu$ (achieved by addition of uncharged Dextran to final concentration of 5\%) illustrating the inherent versatility of the cone-plate apparatus. Note the greater dependence of shear stress magnitude on radius, $r$, for large values of $R_{e}$. The weighted averaged values of $R_{e}$ and $\tau_{\mathrm{ss}}$ for the case of a tissue culture plate with an effective culture area of maximal radius of $4 \mathrm{~cm}$ are 21.5 and $21.4 \mathrm{dyn} / \mathrm{cm}^{2}$ for set $\mathrm{A}, 0.7$ and $14.7 \mathrm{dyn} / \mathrm{cm}^{2}$ for set $\mathrm{B}$, and 0.04 and $49 \mathrm{dyn} / \mathrm{cm}^{2}$ for set C respectively. It is important to note that the values of $\tau_{s s}$ for set $A$ are less accurate than those for $B$ and $C$ given the large values of $R_{e}$ involved in the turbulent flow regime.

contact with the cell growth surface. In order to overcome this problem, the device is designed to insure free sliding of the cone and its shaft assembly within the inner race of the bearings and to include a spring acting in compression to press the cone at its tip. Figure 5 illustrates the importance of these features. Measurement of the depth of the TCP bottom surface reveals significant difference from the perfectly-flat plate when left unrestrained (Figure 6, open circles). In contrast, when the cone is in position, and is brought to bear through its tip on the tissue culture surface, the restrained plate becomes significantly flatter (Figure 6, solid circles). This is a critical point, especially at small cone angles, and allows the use of cones with $\alpha$ as low as $0.5^{\circ}$ without exceeding $10 \%$ of the gap corresponding to a perfectly-flat plate.

\section{Testing of fluid mechanical properties}

To insure that device performance is consistent with theoretical predictions, flow line analysis is carried out by slow infusion of a fine jet of ink through a narrow port flush with the surface of the tissue culture plate at a fixed radius $r=r^{\prime}$ while rotational velocity, $\omega$ and viscosity of the media, $\mu$, are varied to span values of $R_{e}$ from 0.03 to 3 . The angle $(\phi)$ formed between the ink line and the tangent is measured (Figure 7A). Figure $7 \mathrm{~B}$ shows both the theoretical prediction [30] and the experimental measurements, indicating a close correlation between measured and predicted $\phi$. These results demonstrate that the computations used in predicting shear stress

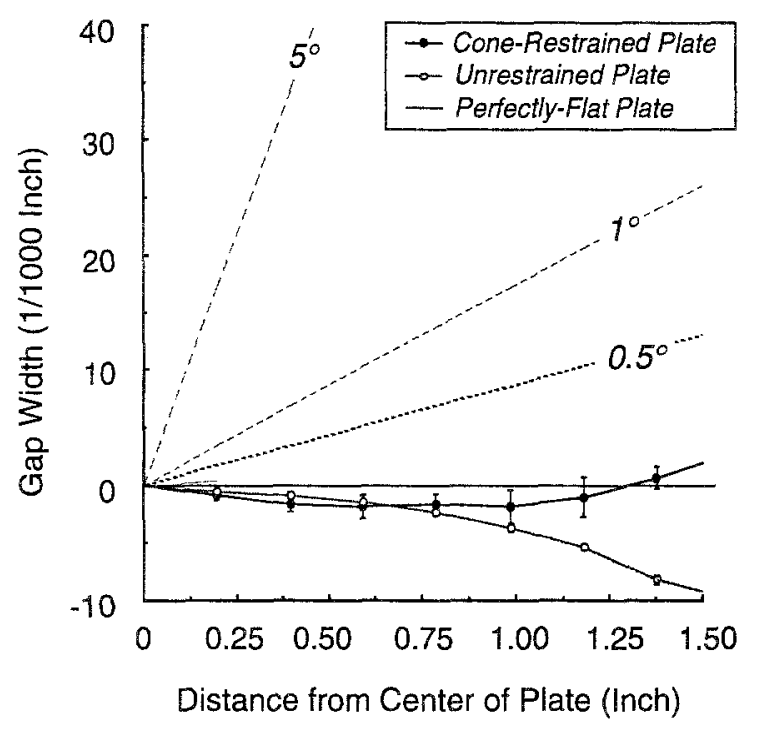

Figure 6. Critical importance of the spring loaded/sliding shaft design in permitting the use of standard commercially available tissue culture plates. The UHMW polyethylene tip at the center of the cone is pressed down by a central spring, thereby flattening the tissue culture plate surface to approximate a flat surface. Note significant improvement of flatness in the cone-restrained case (closed circles) when compared to the unrestrained tissue culture plate surface (open circles, averaged of 3 different samples of Falcon model, No. 3003 plates). Straight lines illustrate the gap, $h(r)$, between an ideal flat surface and the cone surface for angle $\alpha$ of $0.5^{\circ}, 1^{\circ}$ and $5^{\circ}$ at various values of $r$. The actual gap is obtained by measuring the difference between the ideal cone gap width and the measured gap width of the cone-restrained plate. 

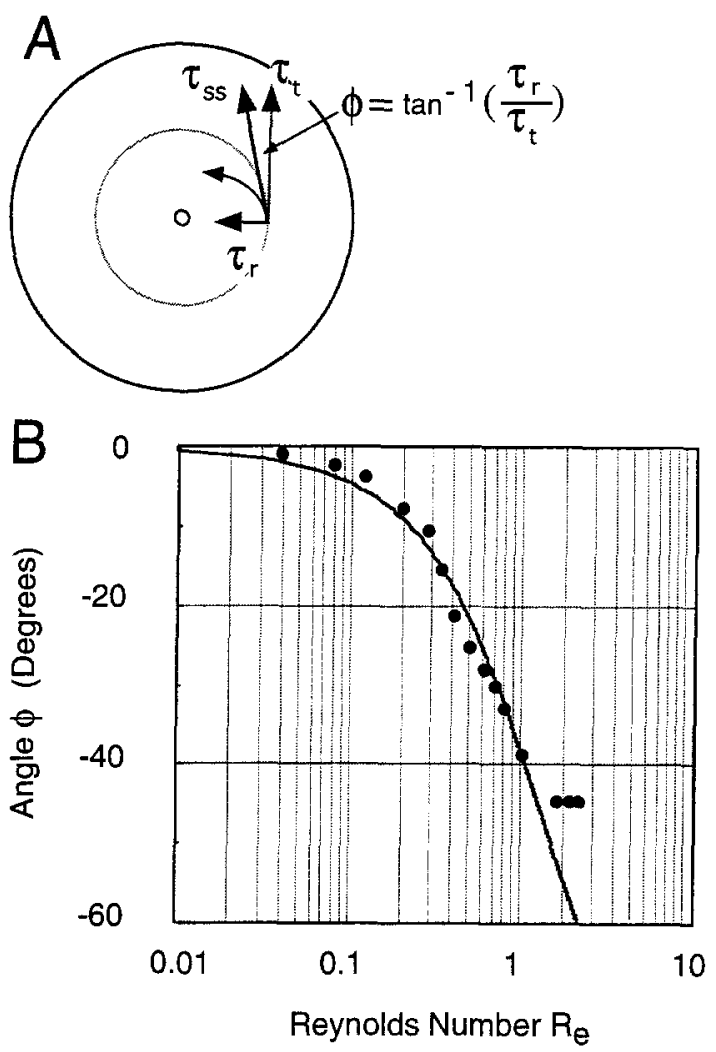

Figure 7. Ink-line flow analysis performed by passing a fine jet of ink at the surface of the plate using a cone of $1^{\circ}$ angle rotating at various velocities $\omega$ and medium viscosities $\mu$, resulting in increasing values of $R_{\mathrm{e}}$. (A) To a given value of $R_{e}$ corresponds a theoretically predicted value of the angle $\phi$ formed between shear stress vector at the surface consisting both of primary flow component, $\tau_{\mathrm{t}}$ (tangent direction), and a secondary flow component, $\tau_{\mathrm{r}}$ (radical direction). (B) Experimental measurements of the angle $\phi$, using the $1^{\circ}$ cone for $15 \mathrm{~cm}$ tissue culture plate, shown for various values of $R_{e}(d o t)$; these compare favorably with the predicted values (line) for values of $R_{e}$ up to $\sim 1-2$, indicating good correlation between predicted and actual values of shear stress.

magnitude and Reynolds number are correct to within approximately $5-10 \%$ up to $\mathrm{R}_{\mathrm{c}}$ of about 2 .

\section{Control of cone rotation velocity and step response} to external velocity command

Since the device is also intended to allow application of pulsatile and reversing shear stress, it is important to assess the ability to control cone rotation in the fully-assembled and operational setting. To achieve that end and further characterize the operation of the DC-motor controller, we analyzed the temporal response of cone velocity to a unit step command of either 3 or $6 \mathrm{rev} / \mathrm{s}$ (final velocity). Figure 8A illustrates the response for both the small size cone designed for use with $10 \mathrm{~cm}$, and the large one for the $15 \mathrm{~cm}$ TCP. Both responses exhibited a delay, $t_{d}$, during which cone velocity appeared to increase in a linear fashion to its final value. The value of $t_{d}$ to a step input of 3 and $6 \mathrm{rev} / \mathrm{s}$ was measured at 24 and $68 \mathrm{~ms}$ for the small cone and 93 and $183 \mathrm{~ms}$ for the large cone respectively. Figure 8B (top) illustrates the capability of driving the controller with a signal generator to achieve pulsatile and reversing cone velocity waveforms. In addition, the controller accepts inputs from the D/A board of a personal computer, which allows the user to arbitrarily choose a velocity waveform (Figure $8 \mathrm{~B}$, bottom).

\section{Dynamic frequency response analysis}

To better define the dynamic characteristics of the fully-configured cone-plate apparatus, a frequency response analysis of the cone velocity using a sinusoidal input signal was undertaken. The results appear in Figure 9A for the $10 \mathrm{~cm}$ plate system using Falcon model, No. 3003 TCP, and demonstrate a flat response (near to $1: 1$ gain vale) up to a stimulus frequency to $10 \mathrm{~Hz}$ and $8 \mathrm{~Hz}$ for a velocity of 3 and $6 \mathrm{rev} / \mathrm{s}$ respectively. When using the larger $15 \mathrm{~cm}$ TCP setup, with the associated increase in size and inertia of the larger cone, the breakaway frequency is considerably lower at 5 and $3 \mathrm{~Hz}$ for 3 and $6 \mathrm{rev} / \mathrm{s}$ respectively. It is worthwhile to note the slight frequency-dependent increase in gain as one approaches the breakaway frequency value. Furthermore, the system appears not to be linear, and demonstrates both amplitude- and frequency-dependence. Nonetheless the user can safety assume linear response when using stimulus frequencies of less than $3 \mathrm{~Hz}$ and considerably higher when using the smaller cone at lower speeds. Since a frequency of $3 \mathrm{~Hz}$ corresponds to 180 heart beats/min, the system can under worst circumstances simulate physiological flow conditions at very high heart rate.

\section{Fluid mechanical considerations when using a dynamic stimulus}

When using the device with time-varying stimuli, it is important to insure that cone acceleration is kept low enough to permit sufficient flow development and shear transmission to the plate below. This problem can be approximated (for small cone angles) by that of the suddenly-accelerated plate [23]. The solution to this problem requires that the following condition be satisfied in order for the difference between the velocity profile and its asymptotic value be less than $1 \%$ :

$$
t_{0}>\frac{0.25 h(\mathrm{r})^{2}}{v},
$$

where $t_{0}$ is the time of development, $h(\mathrm{r})$ is the gap between the plate and the cone surface at a given radius and $v$ is the kinematic viscosity. An illustrative calculation for a cone of $1^{\circ}$ and maximum radius $\mathrm{r}_{0}$ of $4 \mathrm{~cm}$ using regular DME with $10 \%$ calf serum would yield $t_{0}>4 \mathrm{~ms}$. This is clearly negligible when using a sinusoidal waveform of frequency $90 / \mathrm{min}$ (Figure 8B, pulsatile) with a corresponding period of $\mathrm{T}=667 \mathrm{~ms}$. 


\section{A Step response}

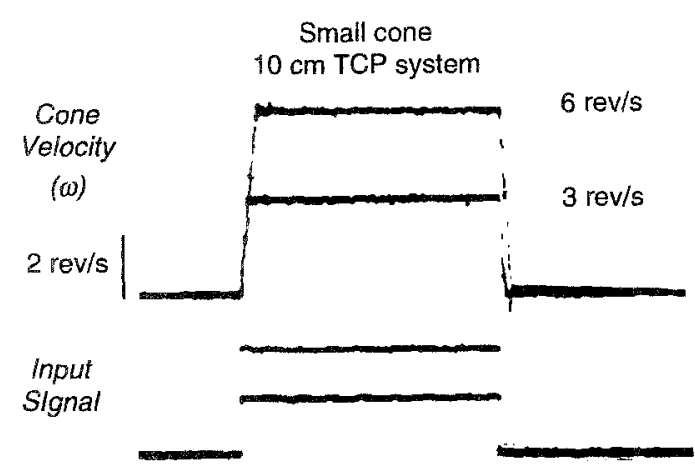

$\overline{0.25}$

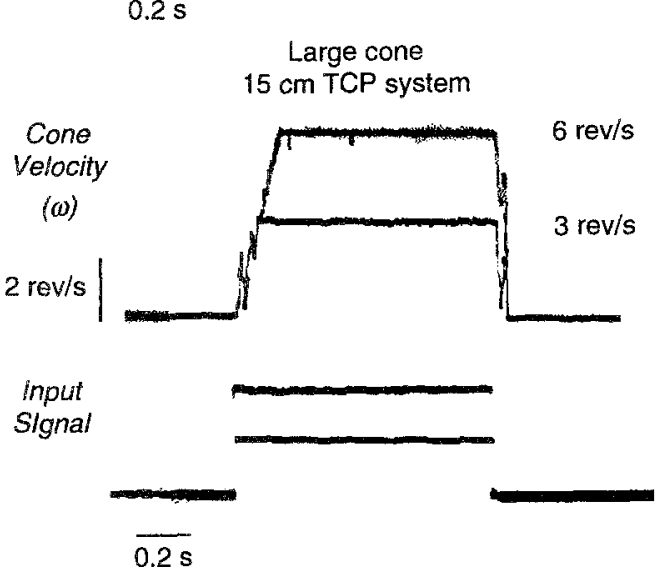

B
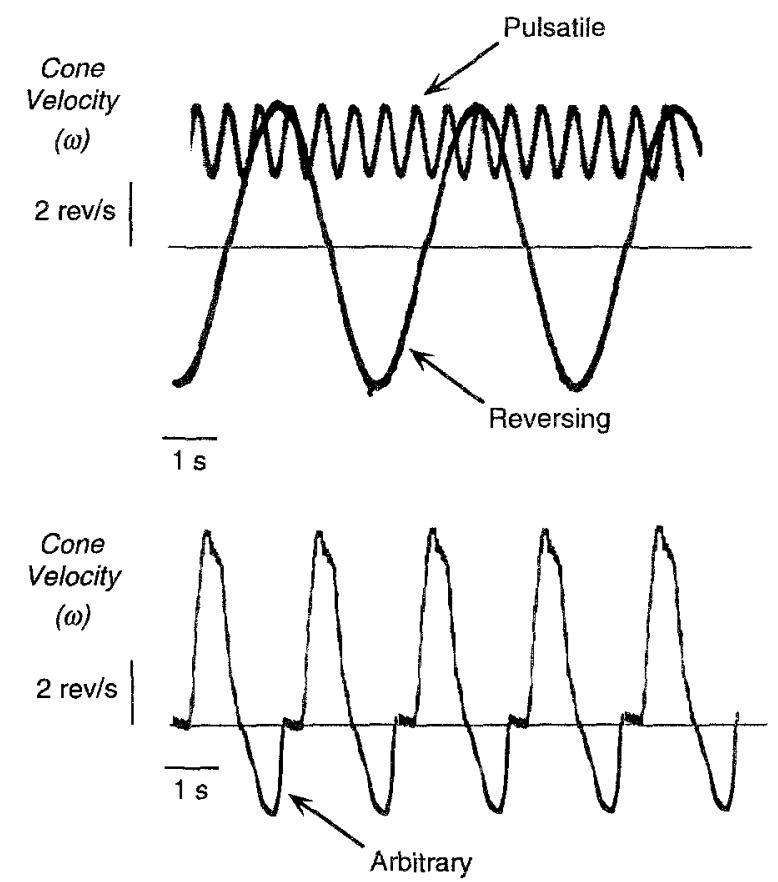

Figure 8. (A) Determination of the response of cone velocity, $\omega$, in response to a step input at the DC-motor controller to a final rotational velocity of 3 and $6 \mathrm{rev} / \mathrm{s}$. Top set of traces illustrate the response for the small size cone used with $10 \mathrm{~cm}$ tissue culture plates (Falcon model, No. 3003), with a delay, $t_{\mathrm{d}}$, to reach final velocity of 3 and $6 \mathrm{rev} / \mathrm{s}$ being 24 and $68 \mathrm{~ms}$ respectively. The bottom set for the large size cone used with $15 \mathrm{~cm}$ tissue culture plates (Falcon model, No. 3025 ) requires 93 and $183 \mathrm{~ms}$ to achieve 3 and $6 \mathrm{rev} / \mathrm{s}$ from a full stop respectively.

(B) Typical cone velocity tracings in response to an input signal from an external source to the DC-motor controller; top tracing shows pulsatile waveform varying from 3 to $5 \mathrm{rev} / \mathrm{s}$ at a frequency of $90 / \mathrm{min}$ and reversing waveform varying from $5 / \mathrm{s}$ in one direction to the other at a frequency of $15 / \mathrm{min}$, while bottom tracing shows the velocity response to a computer-generated arbitrary waveform (output from D/A converter to the DC-motor controller) with a biphasic peak and a reversing component, at a frequency of approximately $30 / \mathrm{min}$.

In the turbulent regime $\left(R_{e}>4\right)$, it is possible to estimate the order of magnitude of the turbulent eddies generated by computing the Kolmogorov scale; this value can be computed in the cone-plate configuration to be approximately equal to $0.1 * h(\mathrm{r})$, or $10 \%$ of the local gap between the cone and the plate $[28,36]$. The size of the turbulent eddies gives an approximation of the size of the local currents generated at the level of the cells inside the tissue culture plate. Such an estimate may be helpful in comparing the nature of the applied shear stimulus to cellular dimensions or to values of turbulent eddies encountered in certain pathological situations in vivo.

Long-term culture and biological response of $B A E$ cells to fluid shear stress

To verify the long-term survival of cells inside the present device and to insure lack of toxicity of the materials used, BAE cells grown in DME supplemented with $10 \%$ calf serum were exposed to 15 $\mathrm{dyn} / \mathrm{cm}^{2}$ for 24 hours. At the end of the experiment, the supernatant overlying these cells was analyzed for LDH content and compared to total intracellular LDH content. The result from triplicate determinations indicate virtually no difference between $\mathrm{BAE}$ cells exposed to 24 hours of shear and control cells kept under static conditions, underscoring the lack of injury (Table 1). Further experiments were conducted where BAE cells were exposed to up to 96 hours of constant fluid shear of $15 \mathrm{dyn} / \mathrm{cm}^{2}$ in the steady laminar regimen and showed continual integrity of the BAE monolayer with frank alignment of the cells in the direction of the flow line [33]. 
A

Small cone $10 \mathrm{~cm}$ TCP System

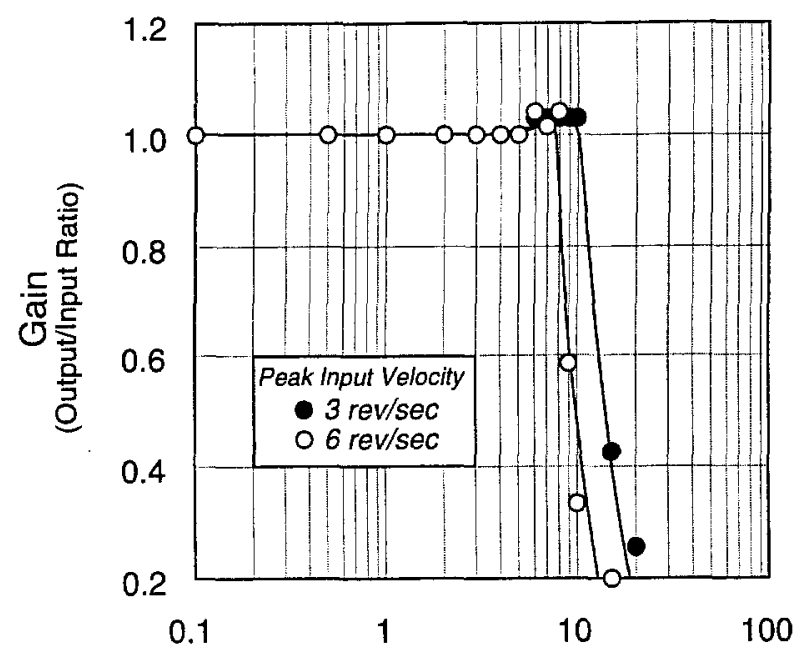

Frequency of Stimulus $(\mathrm{Hz})$

B

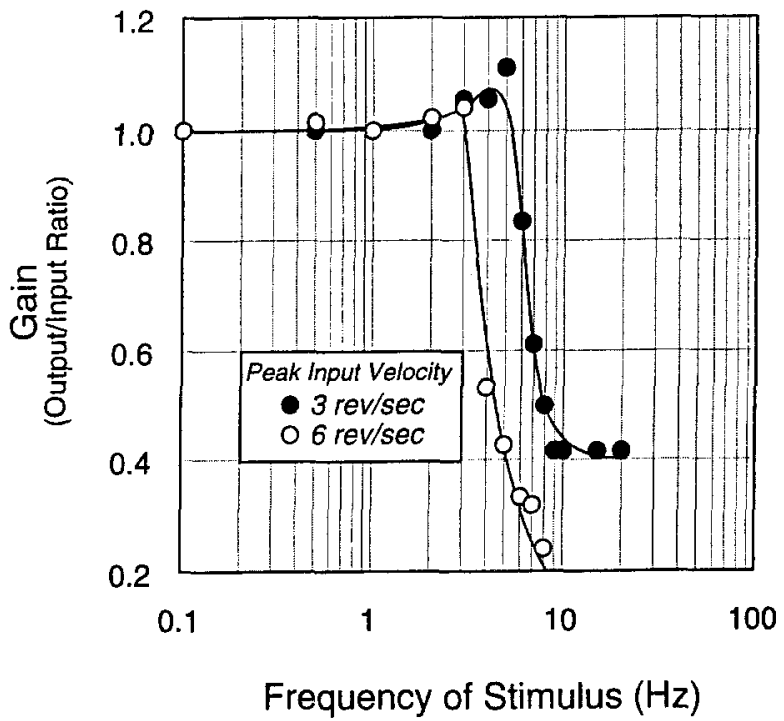

Figure 9. Dynamic response analysis of the operational cone-plate apparatus. A sinusoidal wave was used as input to the motor controller and the rotational velocity of the cone $(\omega)$ measured using a storage oscilloscope at increasing frequency values. A gain value was defined as the ratio of the peak rotational velocity to the peak of the input wave (either $3 \mathrm{rev} / \mathrm{s}$ or $6 \mathrm{rev} / \mathrm{s}$ ).

(A) Frequency response analysis for the $10 \mathrm{~cm}$ TCP (Falcon model, No. 3003) showing a frequency dependent fall-off at frequencies above $10 \mathrm{~Hz}$ and $8 \mathrm{~Hz}$ for peak input velocities of 3 and $6 \mathrm{rev} / \mathrm{s}$ respectively. Note the magnitude-dependent decrease in breakaway frequency.

(B) Frequency response analysis for the $15 \mathrm{~cm} \mathrm{TCP}$ (Falcon model, No. 3025) revealing much lower breakaway frequencies of 5 and $3 \mathrm{~Hz}$ respectively. This is to be expected given the larger size and associated higher inertia in the $15 \mathrm{~cm}$ system.
Table 1. Measurement of the release of intracellular lactic dehydrogenase $(\mathrm{LDH})$ into the overlying medium in $\mathrm{BAE}$ cells exposed to $15 \mathrm{dyn} / \mathrm{cm}^{2}$ for shear stress durations of 12 and 24 hours. These values are expressed as a percentage released as a function of the total intracellular LDH content. The results indicate low level of injury in response to shear stress which is not significantly different from static controls.

\begin{tabular}{lll}
\hline Time (hours) & Shear stress (in \%) & Static control (in \%) \\
\hline 0 & $0.00 \pm 0.05$ & $0.00 \pm 0.04$ \\
12 & $0.99 \pm 0.14$ & $1.18 \pm 0.09$ \\
24 & $3.23 \pm 0.35$ & $3.00 \pm 0.25$ \\
\hline
\end{tabular}

Relative advantage of cone-plate versus parallelplate and capillary tube viscometers

The cone-plate viscometer design is inherently flexible and, using the appropriate parameters, allows the exposure of the cells to steady, time- or directionvarying laminar (primary or secondary mode) and turbulent shear stress. It is possible to obtain pulsatile shear stress by introducing a determined wobble between the cone shaft base and the cone $[10,21]$. Given the ability to achieve arbitrary cone rotational velocities, the low values of $t_{\mathrm{d}}$, and the elevated frequency response of the device, the setup readily achieves pulsatile and other time-varying shear stress patterns $[10,23]$. The present design is simple, versatile, and lends itself to the exposure of large samples of adherent cells to shear stress without requiring complex tubing or pump mechanisms that may potentially introduce pressure artifacts [27]. Unlike parallel-plate and capillary tube viscometers that rely on pressure heads or peristaltic pumps to force the fluid over the cell monolayer, the device presented here allows the flow medium to remain at atmospheric pressure. This feature enables one to uncouple the effect of hydrodynamic pressure from that of shear stress, and presents an advantage over the alternative viscometers. Furthermore, by modifying the present device to include hermetic seals around the cone shaft and coverplate, it is possible to pressurize the medium inside the present device, and thus assume independent control over hydrostatic pressure and fluid shear stress. It is important to point out that the present device has some potential disadvantages when compared to parallel-plate and capillary tube viscometers: (1) it is somewhat more complex and time-consuming to build; the shallow angle of the cone requires high precision ball bearings, precision-ground cone shaft, and a low level of machining tolerance for proper alignment and operation, (2) the shear stress and Reynolds number values require more complex computation, and involve numerical integration, (3) the shear stress magnitude exhibits, under certain values of cone angle and rotational velocity, larger variation within the tissue culture plate as a result of the dependence 
on radius, $r$, which is steep at high values of $R_{e}$. There also exist a number of applications such as local whole cell measurement of membrane potential [25] or cell-to-cell measurement of $\mathrm{pH}_{\mathrm{i}}$ [26] or ionic concentrations in which the simplicity of the capillary tube viscometer may be better suited.

\section{Examples of use and flexibility of presented apparatus}

Multiple lines of evidence have implicated mechanical forces of hemodynamic origin in the control of cardiovascular system structure and function. The need to characterize the cellular response of the various components of the heart and vessel wall calls for specialized tissue culture systems in an attempt to reproduce the mechanical stimuli that occur naturally in both physiological and pathological states. The present paper presents in detail the method of construction and testing of an apparatus which is easily integrated into standard tissue culture technique and allows the exposure of adherent cells to well-controlled levels of fluid shear stress. We have confirmed the sound design of this apparatus, the concept of using commercially available TCP, and the construction method used by testing fluid mechanical properties by ink flow line and dynamic frequency response analyses, and lack of cell injury by demonstrating no increase in LDH release when compared to static controls. Using the described apparatus, we have detected supernatant levels of endothelin-1 peptide [10], measured changes in DNA and RNA rates of synthesis [1], reported altered gene expression [10,33] and performed nuclear runoff transcription and transfection assays for promoter analysis of the endothelin-1 gene to shear stress [37]. In summary, the present device is versatile, selfcontained, provides reproducible fluid mechanical properties, and is optimized for the biochemical, molecular and cellular biological study of the response of endothelium and other adherent cells to fluid shear stress.

\section{Acknowledgements}

This work was supported by a Whitaker Foundation Grant to S.I., and by a Johnson \& Johnson Foundation Research Grant (through the Harvard-MIT Health Sciences and Technology Division) and a National Institutes of Health Medical Scientist Training Program (MSTP) Grant to A.M.

\section{Notes on suppliers}

1. Robbins \& Myers/Electrocraft, Eden Prairie, MN, USA

2. Berg, New York, NY, USA

3. Boston Centerless, Malden, MA, USA

4. Modern Plastics, Peabody, MA, USA

5. Barden Bearings, CT, USA
6. Data Translation, Burlington, MA, USA

7. Hitachi Electronics, Japan

8. NF Electronics, Japan

9. International Business Machines, Boca Raton, FL, USA

10. Apple Computer, Cupertino, CA, USA

11. Queue Tissue Culture Systems, Woburn, MA, USA

12. Olympus, Japan

13. Becton Dickinson Labware, Franklin Lakes, NJ, USA

14. Costar Labware, Cambridge, MA, USA

15. Fischer Scientific, Pittsburgh, PA, USA

16. Dow Corning, New York, NY, USA

17. MathSoft, Cambridge, MA, USA

18. Gibco-BRL, Gaithersburg, MD, USA

19. Sigma Chemicals, St Louis, MO, USA

20. Biomedical Technologies, Stoughton, MA, USA

21. Arena Farms, Hopkington, MA, USA

\section{References}

1. Malek AM, Izumo S (1994). Molecular aspects of signal transduction of shear stress in the endothelial cell. J Hypertens 12: 989-999.

2. Davies PF, Tripathi SC (1993). Mechanical stress mechanisms and the cell, an endothelial paradigm. Circ Res 72: 239-245.

3. Sumpio BE, Banes AJ, Link WG, Johnson Jr G (1988). Enhanced collagen production by smooth muscle cells during repetitive mechanical stretching. Arch Surg 123: 1233-1236.

4. Sadoshima J, Izumo S (1993). Mechanical stretch rapidly activates multiple signal transduction pathways in cardiac myocytes: potential involvement of an autocrin/paracrine mechanism. EMBO J 12: 1681-1692.

5. LaBarbera M (1990). Principles of design of fluid transport systems in zoology. Science 249: 992-1000.

6. Remuzzi A, Dewey Jr F, Davies P, Gimbrone Jr MA (1984). Orientation of endothelial cells in shear fields in vitro. Biorheology 21: 617-630.

7. Franke $R$, Gräfe $M$, Schnittler $H$, Seiifge D, Mittermayer C, Drenckhahn D (1984). Induction of human vascular endothelial stress fibers by fluid shear stress. Nature 307: 648-649.

8. Diamond SL, Sharefkin JB, Dieffenbach C, FrasierScott K, McIntire LV, Eskin SG (1990). Tissue plasminogen activator messenger RNA levels increase in cultured human endothelial cells exposed to laminar shear stress. J Cell Physiol 143: 364-371.

9. Hsieh H, Li N, Frangos J (1991). Shear stress increases endothelial platelet-derived growth factor mRNA levels. Am J Physiol 260: H642-H646.

10. Malek A, Izumo S (1992). Physiological fluid shear stress causes down-regulation of Endothelin-1 mRNA in bovine aortic endothelium. Am J Physiol 32: C389-C396.

11. Kamiya A, Bukhari R, Togawa T (1984). Adaptive regulation of wall shear stress optimizing vascular tree function. Bull Math Biol 46: 127-137.

12. Zarins CK, Zatina MA, Giddens DP, Ku DN, Glagov $S$ (1987). Shear stress regulation of artery lumen diameter in experimental atherogenesis. J Vasc Surg 5: 413-420. 
13. Goldsmith HL, Turitto VT (1986). Rheological aspects of thrombosis and haemostasis: Basic principles and applications. Thromb Haem 55: 415-435.

14. Asakura T, Karino T (1990). Flow patterns and spatial distribution of atherosclerotic lesions in human coronary arteries. Circ Res 66: 1045-1066.

15. Shen J, Luscinskas FW, Connolly A, Dewey Jr F, Gimbrone Jr M (1992). Fluid shear stress modulates cytosolic free calcium in vascular endothelial cells. Am J Physiol 262: C384-C390.

16. Geiger RV, Berk BC, Alexander RW, Nerem RM (1992). Flow-induced calcium transients in single endothelial cells: Spatial and temporal analysis. Am J Physiol 262: C1411-1417.

17. Prasad ARS, Logan SA, Nerem RM, Schwartz CJ, Sprague EA (1993). Flow-related responses of intracellular inositol phosphate levels in cultured aortic endothelial cells. Circ Res 72: 827-836.

18. Frangos JA, Eskin SG, McIntire LV, Ives CL (1984). Flow effects on prostacyclin production by cultured human endothelial cells. Science 227: 1477-1479.

19. Wechezak AR, Wight TN, Viggers RF, Sauvage LR (1989). Endothelial adherence under shear stress is dependent upon microfilament reorganization. J Cell Physiol 139: 136-146.

20. Levesque MJ, Nerem RM, Sprague EA (1990). Vascular endothelial cell proliferation in culture and the influence of flow. Biomaterials 11: 702-707.

21. Davies PF, Dewey Jr FC, Bussolari SR, Gordon EJ, Gimbrone Jr MA (1983). Influence of hemodynamic forces on vascular endothelial function, in vitro studies of shear stress and pinocytosis in bovine aortic cells. J Clin Invest 73: 1121-1129.

22. Bussolari SR, Dewey Jr CF, Gimbrone Jr MA (1982). Apparatus for subjecting living cells to fluid shear stress. Rev Sci Instrum 53: 1851-1854.

23. Sutera SP, Nowak MD (1988). A programmable, computer-controlled cone-plate viscometer for the application of pulsatile shear stress to platelet suspensions. Biorheology 25: 449-459.

24. Diamond S, Eskin S, McIntire L (1989). Fluid flow stimulates tissue plasminogen activator secretion by cultured human endothelial cells. Science 243: 1483-1485.

25. Olesen S, Clapham DE, Davies PF (1988). Haemodynamic shear stress activates a $\mathrm{K}^{+}$current in vascular endothelial cells. Nature 331: 168-170.

26. Ziegelstein RC, Cheng L, Capogrossi MC (1992). Flow-dependent cytosolic acidification of vascular endothelial cells. Science 258: 656-659.
27. Sumpio BE, Widmann MD, Ricotta J, Awolesi MA, Watase M (1994). Increased ambient pressure stimulates proliferation and morphologic changes in cultured endothelial cells. J Cell Physiol 158: 133-139.

28. Davies PF, Remuzzi A, Gordon E, Dewey Jr F, Gimbrone Jr MA (1986). Turbulent fluid shear stress induces vascular endothelial cell turnover in vitro. Proc Natl Acad Sci USA 83: 2114-2117.

29. Hsieh H-J, Li N-Q, Frangos J (1993). Pulsatile and steady flow induces c-fos expression in human endothelial cells. J Cell Physiol 154: 143-151.

30. Sdougos HP, Bussolari SR, Dewey Jr CF (1984). Secondary flow and turbulence in a cone-plate device. J Fluid Mech 138: 379-404.

31. Schnittler H-J, Fränke RP, Akbay U, Mrowietz C, Drenckhahn D (1993). Improved in vitro rheological system for studying the effect of fluid shear stress on cultured cells. Am J Physiol 265: C289-C298.

32. Yoshizumi M, Kurihara H, Sugiyama T, Takaku F, Yanagisawa M, Masaki T, Yazaki Y (1989). Hemodynamic shear stress stimulates endothelin production by cultured endothelial cells. Biochem Biophys Res Comm 161: 859-864.

33. Malek AM, Gibbons GH, Dzau VJ, Izumo S (1993). Fluid shear stress differentially modulates expression of genes encoding basic fibroblast growth factor and platelet-derived growth factor B-chain in vascular endothelium. J Clin Invest 92: 2013-2021.

34. Gimbrone Jr MA, Cotran R, Folkman J (1974), Human vascular endothelial cells in culture. J Cell Biol 60: 673-684.

35. Netland PA, Zetter BR, Via DP, Voyta JC (1985). In situ labelling of vascular endothelium with fluorescent acetylated low density lipoprotein. Histochemical $\mathbf{J}$ 17: 1309-1320.

36. Corrsin S (1959). Outline of some topics in homogeneous turbulent flow. J Geophys Res 64: 2134-2150.

37. Malek AM, Greene AL, Izumo S (1993). Regulation of endothelin-1 gene by fluid shear stress is transcriptionally mediated and independent of protein kinase C and CAMP. Proc Natl Acad Sci USA 90: 5999-6003.

Address for correspondence: Adel M. Malek, MD, $\mathrm{PhD}$, Department of Neurosurgery, Brigham \& Women's Hospital/Children's Hospital, 75 Francis Street, Boston, MA 02115, USA

Phone: (617) 732 6660; Fax: (617) 7324144

E-mail: ammalek@bics.bwh.harvard.edu 